\title{
AUFSÄTZE
}

\section{Das Bundesverfassungsgericht als Gesetzgeber anstelle des Gesetzgebers: Ein kritischer Blick auf das Wahlrechtsurteil vom 25. Juli 2012}

\author{
Volker M. Haug
}

Mit seinem jüngsten Wahlrechtsurteil hat das Bundesverfassungsgericht „tabula rasa“ gemacht und das neue Wahlrecht gleich dreifach für verfassungswidrig erklärt: Der bereits im Urteil vom 3. Juli 2008 beanstandete Effekt des negativen Stimmgewichts bestehe in erheblichem Umfang fort, die neu eingeführten Zusatzmandate seien willkürlich und die Anzahl der auch künftig zu erwartenden (ausgleichslosen) Überhangmandate sei zu hoch. Im letzten Punkt nahm das Gericht zu einem „Wahlrechtsklassiker" Stellung, der schon intensiv das politik- und rechtswissenschaftliche Schrifttum beschäftigt hat ${ }^{1}$; auch die öffentliche Meinung hat sich schon mehrfach - insbesondere nach Wahlen mit zahlreichen Überhangmandaten - über die vermeintliche Ungerechtigkeit der Überhandmandate erregt, ohne dass diese bislang von Karlsruhe an den Pranger der Verfassungswidrigkeit gestellt wurden. Insofern dürfte das nun vorliegende Urteil dem „Mainstream“ der öffentlichen wahlrechtspolitischen Debatte zumindest in weiten Teilen entsprechen. Ob es in allen Punkten auch verfassungsrechtlich überzeugt, steht auf einem anderen Blatt.

\section{Vorgeschichte}

\subsection{Dresdner Nachwahl 2005}

Die Wahlrechtsauseinandersetzung der letzten Jahre nahm in Dresden ihren Anfang. Weil eine Direktkandidatin im Wahlkreis 160 (Dresden I) elf Tage vor der Bundestagswahl 2005 verstarb, wurde gemäß $\$ 43$ Abs. 1 Nr. 2 BWahlG eine Nachwahl nötig, die aus organisato-

1 Dabei überwiegen die kritischen Stimmen zu den Überhangmandaten, vgl. Hans-Heinrich Trute, in: Ingo von Münch / Philip Kunig (Hrsg.), Grundgesetz Kommentar, München 2012, Art. 38 Rn. 61, der von einer „Verdoppelung der Stimmen“ spricht, weil hier neben der Zweit- auch der Erststimme Mandatsrelevanz zukommt; Hans Meyer, in: Josef Isensee / Paul Kirchhof (Hrsg.), Handbuch des Staatsrechts, Band III, Heidelberg 2005, $\$ 46$ Rn. 45 ff., insbesondere Rn. 47; Joachim Behnke, Überhangmandate und negatives Stimmgewicht: Zweimannwahlkreise und andere Lösungsvorschläge, in: ZParl, 41. Jg. (2010), H. 2, S. 247 - 260, S. 248; Manfred C. Hettlage, Mehr Stücke als Kuchen - Also ist das Parlament kleiner als die Summe seiner Mitglieder, in: ZRP 2012, S. 87; Heiko Holste, Die Reform des Bundestagswahlrechts: Wiedervorlage in Karlsruhe, in: NVwZ 2012, S. 8. Im bisherigen Umfang gerechtfertigt sind die Überhangmandate nach Hans H. Klein, in: Theodor Maunz / Günter Dürig (Hrsg.), Grundgesetz Kommentar, Loseblattsammlung, München 2012, Art. 38 Rn. 130a; gänzlich unproblematisch bei Franz Urban Pappi / Michael Herrmann, Überhangmandate ohne negatives Stimmgewicht: Machbarkeit, Wirkungen, Beurteilung, in: ZParl, 41. Jg. (2010), H. 2, S. 260 - 278, S. 271. 
rischen Gründen erst 14 Tage nach der Hauptwahl durchgeführt werden konnte. Weil das Wahlergebnis in den 298 anderen Wahlkreisen nicht bis dahin geheim zu halten war ${ }^{2}$, trat der Effekt des negativen Stimmgewichts in das öffentliche Bewusstsein. Ein „normal“ gutes Zweitstimmenergebnis für die CDU in Dresden (wie bei der Wahl 2002) hätte in der bundesweiten Verteilung der Mandate unter den CDU-Landesverbänden eine Verschiebung eines Mandats von einem anderen Bundesland nach Sachsen bewirkt; da die sächsische CDU aber bereits mehr Direktmandate errungen hatte, als ihr nach der Zweitstimmenverteilung zustanden (Überhangmandate), hätte die CDU-interne Verschiebung keinen tatsächlichen Zugewinn in Sachsen (wegen Abschmelzens eines Überhangmandats), sondern nur den Verlust in dem anderen Bundesland zur Folge gehabt. ${ }^{3}$ Dieses Paradoxon, dass ein Mehr an Zweitstimmen zu einem Verlust eines Bundestagsmandats der betreffenden Partei führen kann, wird als „Effekt des negativen Stimmgewichts“ (oder „inverser Erfolgswert“) bezeichnet. Während dieser Effekt wegen der bundesweit zeitgleichen Abhaltung der Wahl für gewöhnlich nicht vorherseh- und steuerbar (aber oft vorhanden) ist, war er in Dresden vor der Nachwahl bekannt, was zu einem erheblichen taktischen Abstimmungsverhalten geführt hat. ${ }^{4}$

\subsection{Die Entscheidung des BVerfG zum Effekt des negativen Stimmgewichts}

Wenig erstaunlich wurde dieser nun offenkundig gewordene Effekt des negativen Stimmgewichts durch Wahlprüfungsbeschwerden angegriffen. Das BVerfG stellte daraufhin in seiner Entscheidung vom 3. Juli 2008 fest, dass der Effekt des negativen Stimmgewichts gegen die Gleichheit und die Unmittelbarkeit der Wahl verstoße. So verlange der Wahlrechtsgleichheitssatz zwar nicht, „dass sich ... für jeden Wähler die ihm gewährleistete gleiche Erfolgschance auch als exakt, verhältnismäßiger' Stimmerfolg realisiert haben muss“, doch sei die Erfolgswertgleichheit jedenfalls dann „in eklatanter Weise“ verletzt, „wenn die beabsichtigten positiven Wirkungen der Stimmabgabe in ihr Gegenteil verkehrt werden"5. Auch die Erfolgschancengleichheit sah das BVerfG verletzt: Zwar wäre eine Wahlrechtskonstellation möglich, bei der manche Stimmen keine Wirkung entfalten (zum Beispiel die Minderheitsstimmen beim Mehrheitswahlrecht), aber keine, bei der positive Stimmen eine negative Wirkung entfalten („zu einem Mandatsverlust beitragen“) können. ${ }^{6}$ Schließlich hat das

2 Zutreffend Helge Sodan / Sebastian Kluckert, Rechtsprobleme durch die Nachwahl zum Deutschen Bundestag, in: NJW 2005, S. 3241, S. 3244, die eine Geheimhaltung „,weder praktisch noch rechtlich" (Verstoß gegen das im Demokratieprinzip wurzelnde Transparenzgebot) für möglich halten.

3 Konkret ging es um das dritte Mandat für die CDU Saarland, das bei einem Überschreiten von 41.226 CDU-Zweitstimmen für die CDU im Wahlkreis 160 als elftes Zweitstimmenmandat nach Sachsen gewandert wäre; wegen der 13 Direktmandate wären dann in Sachsen nur zwei statt drei Überhangmandate angefallen, vgl. http://www.wahlrecht.de/bundestag/2005/nachwahl-dresdenspezial.html (Abruf am 3. August 2012).

4 Die CDU erzielte infolge dessen ein „schlechtes“ Zweitstimmenergebnis von 38.208 Zweitstimmen (2002: 49.638) und konnte so das dritte Zweitstimmenmandat im Saarland beanspruchen, vgl. http://www.bundeswahlleiter.de/de/bundestagswahlen/BTW_BUND_05/ergebnisse/wahlkreisergebnisse/114/wk160/wk_tabelle_160.html (Abruf am 3. August 2012).

6 Ebenda, S. 300 f., Rn. 106. 
BVerfG dem Grundsatz der Unmittelbarkeit der Wahl ein Transparenzgebot entnommen, wonach der Wähler erkennen können muss, „wie sich die eigene Stimmabgabe auf Erfolg oder Misserfolg der Wahlbewerber auswirken kann“, also „ob sich seine Stimme stets für die zu wählende Partei und deren Wahlbewerber positiv auswirkt, oder ob er durch seine Stimme den Misserfolg eines Kandidaten seiner eigenen Partei verursacht ${ }^{\text {"7 }}$. Genau daran fehle es beim Effekt des negativen Stimmgewichts.

Obwohl das BVerfG die Mandatsrelevanz des Wahlrechtsfehlers bejahte, hob es die Bundestagswahl 2005 nicht auf ${ }^{8}$, sondern verpflichtete den Bundesgesetzgeber zu einer gesetzlichen Neuregelung. Wegen der Komplexität des Wahlrechts, der Dauer von Gesetzgebungsverfahren und der relativen zeitlichen Nähe zur nächsten Bundestagswahl im Herbst 2009 räumte das Gericht dem Gesetzgeber eine vergleichsweise großzügige Frist bis zum 30. Juni 2011 ein. ${ }^{9}$ Da das Paradoxon einer Wirkung von Stimmen entgegen ihrer objektiven politischen Absicht schlechterdings nicht zu rechtfertigen ist, hat das Verdikt des BVerfG zum Effekt des negativen Stimmgewichts zu Recht allseitige Zustimmung erfahren, während die Nichtaufhebung der Wahl und insbesondere die Inkaufnahme einer weiteren Bundestagswahl auf der Grundlage eines für verfassungswidrig erkannten Wahlrechts auch auf kritische Stimmen stieß. ${ }^{10}$

Bemerkenswert ist auch, dass das BVerfG den engen Zusammenhang zwischen Überhangmandaten und dem Effekt des negativen Stimmgewichts im alten Wahlrecht klar konstatiert hat, ohne aber auch nur einen Fingerzeig dazu zu geben, wie es zum „Problem“ der Überhangmandate steht. Angesichts der gewöhnlichen Großzügigkeit des Gerichts mit obiter dicta einerseits und der Bedeutung der Überhangmandate für das neu zu schaffende Wahlrecht andererseits ist das nicht zuletzt vor dem Hintergrund der jetzigen Entscheidung und der noch zu erörternden Fürsorge für den Gesetzgeber eher erstaunlich.

\subsection{Umsetzung des Auftrags des BVerfG durch den Gesetzgeber}

Weder vor noch nach der Bundestagswahl 2009 wollte eine parteiübergreifende Verständigung auf ein neues Wahlrecht gelingen. Kernpunkt des Konflikts waren die ausgleichslosen Überhangmandate, die die Union auch künftig ermöglichen wollte. Bündnis 90/Die Grünen und Die Linke wollten die (internen) Überhangmandate einer Partei von den bundesweit erzielten Zweitstimmenmandaten dieser Partei abziehen und damit faktisch mit den Listenmandaten anderer Landesverbände verrechnen. ${ }^{11}$ Die SPD trat demgegenüber dafür ein, die Überhangmandate zwar beizubehalten, aber durch Ausgleichsmandate für die anderen Par-

7 Ebenda, S. 307 f., Rn. 125 - 127.

8 Ebenda, S. 310 ff., Rn. 130 - 139, insbesondere Rn. 138.

9 Ebenda, Ziffer 2 des Urteilstenors und S. 314 ff., Rn. 141 - 144.

10 Insoweit kritisch Gerald Roth, Negatives Stimmgewicht und Legitimationsdefizite des Parlaments, in: NVwZ 2008, S. 1199; dagegen zustimmend - auch zur Novellierungsfrist - Michael König, Paradoxie des negativen Stimmgewichts, in: ZG 2009, S. 177, S. $193 \mathrm{ff}$.

11 Vgl. Text und Begründung von $\$ 7$ a Abs. 7 BWahlG-E des Gesetzentwurfs der Abgeordneten Volker Beck u.a. und der Fraktion Bündnis 90/Die Grünen vom 11. Februar 2009, BT-Drs. 16/11885, S. 3, S. 5; wortgleich $\$ 7$ a Abs. 7 BWahlG-E des Gesetzentwurfs der Abgeordneten Halina Wawzyniak u.a. und der Fraktion Die Linke vom 25. Mai 2011, BT-Drs. 17/5896, S. 7, S. 12. 
teien den parteipolitischen Zweitstimmenproporz wiederherzustellen. ${ }^{12}$ Beide Vorschläge hatten ihre Tücken: Entweder führten sie zu einer erheblichen weiteren Verzerrung der ohnehin schon durch die Überhangmandate disproportionalen Landesgruppenstärken innerhalb der davon betroffenen Fraktionen (Vorschlag Bündnis 90/Die Grünen und Die Linke) oder aber zu einer potentiell erheblichen Ausweitung der Sitzzahl des Bundestages unter Beeinträchtigung der Arbeitsfähigkeit des Plenums (SPD-Vorschlag, der sich deshalb im Vorblatt für eine Reduzierung der Direktmandate und damit der Wahlkreise in Deutschland ausspricht). ${ }^{13}$ Begleitet wurden diese politisch divergierenden Lösungsansätze von einer äußerst intensiven Debatte in Politik- und Rechtswissenschaften, woraus auch zahlreiche Vorschläge - bis hin zu weitreichenden Systemänderungen des Wahlrechts - hervorgingen. ${ }^{14}$

Neben dieser Meinungsvielfalt ist anscheinend auch koalitionsinterner Klärungsbedarf dafür verantwortlich zu machen, dass der Gesetzentwurf von CDU/CSU und FDP erst am 28. Juni 2011 als Drucksache ausgegeben und am 30. Juni 2011 - dem Tag des Fristablaufs - in erster Lesung behandelt wurde. ${ }^{15}$ Dieser Gesetzentwurf, der im Wesentlichen unverändert am 29. September 2011 mit den Stimmen der Koalition gegen die Opposition verabschiedet wurde, versteht sich als „minimalinvasiver Eingriff“ in das Wahlrecht, der in Umsetzung des BVerfG-Auftrags allein zum Ziel hat, den Effekt des negativen Stimmgewichts faktisch auszuschließen. ${ }^{16}$ Hierfür soll die als Hauptursache des Problems angesehene Verbindung von Landeslisten ausgeschlossen und die Ober- und Unterverteilung „umgedreht“ werden: Während bisher die 598 Ausgangsmandate zunächst auf die Parteien (mit mindestens fünf Prozent der Zweitstimmen oder drei Grundmandaten) und anschließend innerhalb jeder Partei auf ihre Landesverbände verteilt wurden, sollen jetzt die 598 Ausgangsmandate zunächst (nach Wählerzahl) auf die einzelnen Länder verteilt werden, bevor innerhalb eines jeden Landeskontingentes eine Verteilung auf die einzelnen Parteien erfolgt. Damit werden die innerhalb einer Partei zuvor möglichen „Mandatswanderungen“ zwischen Landesverbänden mit und ohne Überhangmandate, die zum Effekt des negativen Stimmgewichts führen, ausgeschlossen. Weil mit der neuen Oberverteilung auf 16 Länder auch 16 fache Rundungsverluste verbunden sind, sollen die deswegen nicht mehr mandatsrelevant gewordenen (positiven) Reststimmen jeder Partei länderübergreifend addiert und bei Erreichen der durchschnittlichen Stimmenzahl pro Zweitstimmenmandat als Zusatzmandat vergütet werden. Dieses 19. Änderungsgesetz zum BWahlG stand nun im abstrakten Normenkontrollverfahren auf Antrag der Bundestagsfraktionen von SPD und Bündnis 90/Die Grünen,

12 Vgl. Text und Begründung von $\$ 4$ Abs. 4 BWahlG-E des Gesetzentwurfs der Fraktion der SPD vom 24. Mai 2011, BT-Drs. 17/5895, S. 3, S. 5 f.

13 Diesem Problem trägt das „Anpassungsmodell“ von Daniel Lübbert / Felix Arndt / Friedrich Pukelsheim, Proporzwahrende Anpassung der Bundestagsgröße - ein Lösungsvorschlag für das Problem der negativen Stimmgewichte bei Bundestagswahlen, in: ZParl, 42. Jg. (2011), H. 2, S. $426-435$, mit einer moderateren Sitzzahlerhöhung teilweise Rechnung.

14 Vgl. Wolfgang Schreiber, Das Neunzehnte Gesetz zur Änderung des Bundeswahlgesetzes vom 25. November 2011 - Ein Trauerspiel in vier Akten, in: DÖV 2012, S. 125, S. 127 f.; siehe etwa Manfred C. Hettlage, Das Abgeordneten-Wahlrecht des Bundes ist nicht länger zu halten, in: ZRP 2011, S. 1; Joachim Behnke, Ursachen und Wirkungen - Überlegungen zur Beibehaltung der Überhangmandate im neuen Wahlgesetz, in: ZParl, 43. Jg. (2012), H. 1, S. 170 - 184, S. 180 ff..

15 Vgl. Wolfgang Schreiber, a.a.O. (Fn. 14), S. 127.

16 Vgl. Einbringungsrede des Abgeordneten Günter Krings (CDU/CSU) vom 30. Juni 2012, Plenarprotokoll 17/117, S. 13487 B. 
im Organstreitverfahren auf Antrag der Partei Bündnis 90/Die Grünen sowie infolge von 3.063 Verfassungsbeschwerden von Einzelpersonen auf dem Prüfstand des Bundesverfassungsgerichts.

\section{Das Urteil des BVerfG vom 25. Juli $2012^{17}$}

Das neue Wahlrecht verstößt aus Karlsruher Sicht gleich dreifach gegen das Grundgesetz. Der Effekt des negativen Stimmgewichts, dessen Wahrscheinlichkeit das BVerfG nicht reduziert sieht, verletzt - wie bereits 2008 festgestellt - die Grundsätze der gleichen und der unmittelbaren Wahl gemäß Art. 38 Abs. 1 GG. Die Zusatzmandate und eine zu hohe Zahl an Überhangmandaten verstoßen gegen den Grundsatz der gleichen Wahl gemäß Art. 38 Abs. 1 GG. Alle drei Regelungen sind zudem mit dem Grundsatz der Chancengleichheit der politischen Parteien gemäß Art. 21 Abs. 1 GG unvereinbar, was das Gericht im Hinblick auf die Beteiligung politischer Parteien an diesem Verfahren (Bündnis 90/Die Grünen als Antragstellerin und die NPD als sonstige Beteiligte) besonders betont.

2.1. Keine Reduzierung der Wahrscheinlichkeit des Effekts des negativen Stimmgewichts

\section{(a) Maßstab}

Das BVerfG wirft dem Gesetzgeber vor, die „Hausaufgaben“ aus der Entscheidung von 2008 mit dem 19. Änderungsgesetz zum BWahlG nicht ordnungsgemäß erledigt zu haben. Dabei war die Verpflichtung, ein verfassungsgemäßes Wahlrecht zu schaffen, nicht auf die zwingend vollständige Beseitigung des Effekts des negativen Stimmgewichts gerichtet; vielmehr hat das BVerfG in seiner Entscheidung von 2008 eine geringe Rest-Wahrscheinlichkeit zugelassen. Danach können derartige paradoxe Rechtswirkungen „bei der verfassungsrechtlichen Bewertung des Wahlrechts vernachlässigt werden“, wenn sie nur in „seltenen und unvermeidbaren Ausnahmefällen“ auftreten können. ${ }^{18}$

\section{(b) Argumentation des BVerfG}

Aus Karlsruher Sicht hat die Novellierung die Gefahr, dass der Effekt des negativen Stimmgewichts eintritt, gar nicht reduziert - vielmehr liege die Wahrscheinlichkeit dafür „mindestens in etwa der gleichen Größenordnung “ wie beim alten Wahlrecht. ${ }^{19}$ Lediglich die Anknüpfungspunkte seien nun andere: Während zuvor die Möglichkeit zur Listenverbindung im Zusammenwirken mit den ausgleichslosen Überhangmandaten dafür ursächlich war, ist es nun die wählerabhängige Festlegung der einzelnen Landeskontingente in der neuen Oberverteilung (mit Verstärkungswirkung durch die ausgleichslosen Überhangmandate). Dahinter steht folgender Gedankengang: Bleibt eine bestimmte Anzahl von Wählern einer Partei

17 BVerfG, 2 BvF 3/11 vom 25. Juli 2012, http://www.bundesverfassungsgericht.de/entscheidungen/ fs20120725_2bvf000311.html (Abruf am 25. Juli 2012).

18 BVerfGE, Bd. 121, S. 266, S. 301, Rn. 108; S. 308, Rn. 127; bekräftigt in der aktuellen Entscheidung (a.a.O. (Fn. 17), Rn. 94).

19 BVerfG, a.a.O. (Fn. 17), Rn. 95. 
in einem Bundesland zu Hause, kann dies zur Folge haben, dass sich die Zahl der auf dieses Land in der Oberverteilung entfallenden Sitze reduziert. Dies muss aber keine negativen Folgen für die Sitzzahl der betreffenden Partei haben, entweder weil eine andere Partei den „letzten“ Zweitstimmensitz in diesem Land bekommen hat (und jetzt verliert), oder weil sie in dem betreffenden Bundesland über eine die Zahl der Zweitstimmensitze übersteigende Zahl an Direktmandaten (Überhangmandate) verfügt. Um erneut zu einem Effekt des negativen Stimmgewichts zu gelangen, muss nun aber noch ein zweiter Umstand hinzutreten: Der bei der Oberverteilung „frei gewordene“ und damit einem anderen Land zufallende Zweitstimmensitz müsste gerade in einem solchen Land anfallen, in dem die von der Wählerenthaltung betroffene Partei den höchsten Reststimmenanteil hat und deshalb den nächsten Zweitstimmensitz zugeteilt bekäme. Ist dies der Fall, hat der Stimmenentzug (der im Gegensatz zum alten Wahlrecht nicht zu einem potenziellen Koalitionspartner der betreffenden Partei wandern darf, sondern als Wahlenthaltung zur Reduzierung der Wählerzahl beitragen muss) die paradoxe Wirkung eines Mandatszuwachses für diese Partei. Selbstverständlich lässt sich dieser Effekt auch umgekehrt denken, wonach ein Wähler- und Stimmenzuwachs für eine Partei in einem Land (insbesondere mit Überhangmandaten) zum Verlust eines Sitzes in einem anderen Bundesland und dort auch bei der betreffenden Partei zur Folge haben kann. Zur Untermauerung dieses Effektes führt das Urteil einige Beispiele aus zurückliegenden Wahlen an, in denen bei hypothetischen Stimmenthaltungen entsprechende paradoxe Sitzgewinne möglich gewesen wären. ${ }^{20}$

\section{(c) Bewertung}

So schlüssig dieser Gedankengang ist, so wenig begründet das BVerfG seine Annahme, dass dieser Effekt des negativen Stimmgewichts mit ähnlich hoher Wahrscheinlichkeit eintritt. Dabei sind erhebliche Zweifel angebracht: Während nämlich der Effekt im alten Wahlrecht innerhalb bestimmter (Reststimmen-)Spannen im Zweitstimmenergebnis bei Parteien mit Überhangmandaten regelmäßig - seit 1980 bei jeder Bundestagswahl - aufgetreten ist ${ }^{21}$ (und deshalb auch nicht hinnehmbar war), ist die Wahrscheinlichkeit im neuen Wahlrecht von zwei unterschiedlichen Faktoren abhängig, die nicht miteinander verbunden sind.

Zwar ist dem BVerfG darin zuzustimmen, dass die Plausibilität einer Stimmenthaltung in der Tat mit der des taktischen Stimmverhaltens im alten Wahlrecht vergleichbar ist. ${ }^{22}$ Doch erfordert der erste Faktor des „neuen“ Effekts eines negativen Stimmgewichts, dass sich diese Stimmenreduzierung nicht zulasten der davon betroffenen Partei auswirkt, obwohl die geringere Stimmenzahl mindestens dazu führt, dass sich die Reststimmenzahl dieser Partei im betroffenen Bundesland absolut und im Verhältnis zu den anderen Parteien verschlechtert. Insofern besteht in diesem Fall eine gesteigerte Wahrscheinlichkeit, dass sich der Sitzverlust im Land bei der vom Stimmenentzug betroffenen Partei niederschlägt. Umge-

20 Ebenda, Rn. 88 f.

21 Vgl. im Einzelnen bei http://www.wahlrecht.de/ueberhang/ueberhist.html (Abruf am 5. August 2012).

22 BVerfG, a.a.O. (Fn. 17), Rn. 92; anderer Auffassung die Begründung des Gesetzentwurfs, BT-Drs. $17 / 6290$, S. 8, wonach der kumulative Verzicht auf die Erst- und auf die Zweitstimme (was erforderlich wäre, um die Wählerzahl zu senken) weniger wahrscheinlich sei, als nur die Zweitstimme taktisch einzusetzen. 
kehrt gewendet: Die Wahrscheinlichkeit dafür, dass der mit einem Stimmenentzug für eine Partei verbundene Sitzverlust im Land bei einer der anderen Parteien (ohne Stimmenentzug) zur Mandatsreduzierung führt, ist demgegenüber deutlich geringer. Lediglich beim Vorliegen von Überhangmandaten „schadet“ der wahrscheinliche Verlust eines Zweitstimmenmandats der betroffenen Partei nicht, so dass nur in diesem Fall der erste Faktor regelmäßig eintritt.

Der zweite Faktor, der für den Effekt des negativen Stimmgewichts kumulativ hinzutreten muss, besteht darin, dass sich der Sitzzuwachs gerade bei der vom Stimmenentzug betroffenen Partei auswirkt. Zunächst aber führt der durch die Wählerreduzierung im Land A ausgelöste Sitzverlust nur zu einem Sitzgewinn in einem anderen Land (B), keineswegs aber bei einer bestimmten Partei (anders als im alten Wahlrecht, bei dem es sich um ein durch die Listenverbindung ermöglichtes innerparteiliches Verschiebungsphänomen gehandelt hat). Vielmehr muss „zufällig“ die vom Stimmenentzug im Land A betroffene Partei in dem vom Sitzgewinn betroffenen Land B über den höchsten Reststimmenanteil im Verhältnis zu den anderen Parteien verfügen. Die Wahrscheinlichkeit, dass dieser Fall eintritt, dürfte in einem Fünfparteiensystem durchschnittlich bei circa 20 Prozent liegen. Kombiniert man nun diese gegenüber dem alten Wahlrecht deutlich geringere Wahrscheinlichkeit mit dem erstgenannten Faktor, der außerhalb von Überhangmandaten auch eher als wenig wahrscheinlich anzusehen ist, ergibt sich entgegen der Auffassung des BVerfG eine ganz erheblich reduzierte Wahrscheinlichkeit für den Eintritt des Effekts des negativen Stimmgewichts im neuen Wahlrecht. Die Begründung des Gesetzentwurfs, die diese Konstellation im Blick hat, spricht zutreffend von einer „unwahrscheinlichen Verkettung kumulativer Bedingungen“, die „nicht an der politischen Wirklichkeit orientiert" ${ }^{23}$ sei.

Mit dieser erheblich geringeren Wahrscheinlichkeit ist allerdings noch nichts darüber ausgesagt, ob der Wahlrechtsgesetzgeber dadurch dem vom BVerfG in der Entscheidung von 2008 aufgestellten Maßstab von „seltenen Ausnahmefällen“ hinreichend Rechnung getragen hat. Da der Begriff „selten“ relativ ist, bemisst sich seine Bedeutung aus einer Abwägung zwischen dem demokratischen Prinzip, dass sich Stimmen positiv für ihren Adressaten auswirken müssen, und den Gründen der für diesen „neuen“ Effekt des negativen Stimmgewichts ursächlichen Regelung. Wie auch das BVerfG zutreffend feststellt, liegt diese Ursache in den von der Wählerzahl abhängigen beweglichen Sitzzahlkontingenten der Länder. Hätte man auf die wahlbeteiligungsunabhängige Zahl der Wahlberechtigten oder der Bevölkerung (sic!) je Bundesland abgestellt, wäre aus Karlsruher Sicht jedenfalls dieses Problem zu vermeiden gewesen. ${ }^{24}$ Der Gesetzgeber hat sich jedoch für wählerzahlabhängige Sitzzahlen der Länder entschieden, um vermeidbaren Erfolgswertverzerrungen vorzubeugen; denn bei einer Anknüpfung der Landeskontingente an die Zahl der Wahlberechtigten wären in einem Land mit geringer Wahlbeteiligung entsprechend weniger Zweitstimmen für ein Zweitstimmenmandat erforderlich gewesen als in einem Land mit hoher Wahlbeteiligung. ${ }^{25}$ Das BVerfG erkennt zwar diese Zielsetzung an, hält aber die vom Gesetzgeber vermiedene Erfolgswertverzerrung für „nicht derart gewichtig, dass sie die massive Beeinträchtigung der Wahlrechtsgleichheit und der Chancengleichheit der Parteien durch den Effekt des negati- 
ven Stimmgewichts überwöge“26. Da aber die Wahrscheinlichkeit, dass dieser Effekt eintritt, entgegen der Auffassung des BVerfG durch den Gesetzgeber erheblich reduziert wurde, fehlt es schon an der „massiven“ Beeinträchtigung der verschiedenen Gleichheitsgrundsätze. Vielmehr handelt es sich um zwei verschiedene Übel - die reduzierte Restwahrscheinlichkeit für ein demokratiewidriges Paradoxon einerseits und die Erfolgswertverzerrung bei feststehenden Landeskontingenten andererseits -, zwischen denen der Gesetzgeber eine Auswahlentscheidung getroffen hat. Diesen Beurteilungsspielraum hat das BVerfG aber dem Gesetzgeber verfassungsrechtlich verwehrt, obwohl es in beiden Fällen um Beeinträchtigungen der Erfolgswertgleichheit (und nicht etwa der unbedingten Zählwertgleichheit) geht. Das steht in einem nicht aufgelösten Spannungsverhältnis zum verfassungsgerichtlichen Postulat des weiten Einschätzungs- und Gestaltungsspielraums des Gesetzgebers.

\subsection{Willkürliche Berechnungsgrundlage für die Zusatzmandate}

\section{(a) Argumentation des BVerfG}

Einen weiteren Stein des Anstoßes bilden aus Karlsruher Sicht die so genannten, neu eingeführten Zusatzmandate. So rügt das BVerfG eine doppelte Mandatsrelevanz dieser Reststimmen als ungleiche Behandlung von Wählerstimmen, denn die für eine (in den Bundestag einziehende) Partei in einem Bundesland abgegebenen Zweitstimmen flössen sämtlich in die Unterverteilung in diesem Land ein; auch die (Rest-)Stimmen, die nicht mehr zu einem weiteren Mandat führen (also in den Worten des Gerichts „ohne Erfolgswert bleiben“), seien bei dieser Verteilung „rechtlich gleich“ behandelt worden. Führe man mit den Reststimmen einen "gleichheitswidrig abgegrenzten Teil der Wählerstimmen“ in einem bundesweiten zusätzlichen Zuteilungsverfahren zusammen, räume man ihnen „eine weitere Chance auf Mandatswirksamkeit ein“, was sowohl den Grundsatz der gleichen Wahl als auch die Chancengleichheit der Parteien verletze. Der Gesetzgeber wollte damit die Erfolgswertverzerrung, die durch den 16 fachen Rundungsverlust entsteht, vermeiden. ${ }^{27}$ Das BVerfG erkennt dieses Ziel als denkbaren Rechtfertigungsgrund für die doppelte Mandatsrelevanz dieser Stimmen an, hält aber die konkrete Berechnungsweise zur Erreichung dieses Ziels für ungeeignet, denn ein „Ausgleich der sich aus der vielfachen Sitzzuteilung ... in den Listenwahlkreisen ergebenden kumulierten Rundungsabweichungen" setze voraus, dass nicht nur die positiven Reststimmen aufaddiert und gegebenenfalls in Zusatzmandate umgemünzt werden, sondern zunächst je Partei die positiven und negativen Reststimmen „miteinander verrechnet werden“28. Weil dies aber nicht erfolge, sondern nur Rundungsverluste (also positive Reststimmen) addiert würden, würden die Zusatzmandate nach den Worten des BVerfG „nicht zur Herstellung von Erfolgswertgleichheit, sondern in Abweichung hiervon erzeugt". Weil damit die potentielle Rechtfertigung für die Zusatzmandate entfalle, verstießen diese gegen die Gleichheitsgrundsätze des Wahl- und Parteienrechts. 


\section{(b) Bewertung}

Rundungsverluste treten durch die Abrundungsregel in $\$ 6$ Abs. 2 Satz 3, 1. HS BWahlG dann ein, wenn eine Partei in einem Bundesland nach Anwendung des Divisorverfahrens eine Mandatszahl erreicht, deren erste Nachkommastelle unter fünf liegt (also zum Beispiel bei einer Mandatszahl von 8,4 acht Sitze erhält). Entsprechend umgekehrt führt die Aufrundungsregel in $\$ 6$ Abs. 2 Satz 3, 2. HS BWahlG zu Rundungsgewinnen, wenn die erste Nachkommastelle der Mandatszahl über fünf liegt (also zum Beispiel bei 8,7 neun Sitze zugesprochen werden). Und obwohl Rundungsverluste und -gewinne gleichermaßen wahrscheinlich sind und eine erfolgswertverzerrende Wirkung haben, definiert der Wahlrechtsgesetzgeber im neuen $\$ 6$ Abs. 2a BWahlG ausdrücklich nur die „positiven Abweichungen der auf die Landeslisten entfallenen Zweitstimmen von den im jeweiligen Land für die errungenen Sitze erforderlichen Zweitstimmen" als zusatzmandatsrelevante Reststimmen. Damit verhindert das BWahlG die potentielle Ausgleichswirkung, die Rundungsverluste und -gewinne bei derselben Partei über die 16 Länder hinweg haben können, obwohl die Zusatzmandate nach der Begründung des Gesetzentwurfs gerade dann gerechtfertigt sein sollen, wenn „sich unterschiedlich hohe Zahlen von nicht mehr zu einem Mandat führenden Reststimmen in den 16 Ländern nicht ausgleichen, sondern kumulieren “29. $\$ 6$ Abs. 2a BWahlG ermöglicht dadurch, dass eine Partei mit besonderen Rundungsverlusten in einigen Ländern (ungeachtet eventuell entgegenstehender Rundungsgewinne in anderen Ländern) Zusatzmandate erhalten kann, während eine andere Partei mit einer ähnlichen, aber über die Länder gleichförmiger verteilten Gesamtbilanz diese Mandate nicht erhält, ohne dass dieser in Mandaten zu beziffernde Unterschied irgendeine Rechtfertigung erfährt. Damit liegt in der Tat die vom BVerfG festgestellte Gleichheitsrechtsverletzung vor. Im Hinblick auf die Karlsruher Kritik einer doppelten Mandatsrelevanz von Reststimmen ist jedoch auch festzuhalten, dass Zusatzmandate im Rahmen einer die Rundungsgewinne und -verluste saldierenden Reststimmenregelung bei 16 Listenkreisen nicht nur gerechtfertigt wären, sondern sogar eine Optimierung der Erfolgswertgleichheit und damit aus verfassungsrechtlicher Sicht einen Gewinn darstellen würden. Allerdings müsste eine solche Regelung zur Vermeidung von Erfolgswertverzerrungen dann auch so konsequent sein, dass sie bei einer länderübergreifend entsprechend hohen negativen Reststimmenbilanz einer Partei zu „Abzugsmandaten" führen würde.

\subsection{Ermöglichung von zu vielen Überhangmandaten}

\section{(a) Ursachen und Geschichte der Überhangmandate}

Der Hauptgrund für das Anfallen von Überhangmandaten ist eine Diskrepanz zwischen Erst- und Zweitstimmen einer Partei in einem Bundesland; verstärkend kommt hinzu, dass für ein Direktmandat meist eine relative Mehrheit von 35 bis 40 Prozent ausreichend ist, während die Gesamtzahl der Direktmandate 50 Prozent der 598 Ausgangsmandate beträgt (\$1 Abs. 2 BWahlG). Solange die „großen Volksparteien“ Union und SPD, die faktisch

29 BT-Drs. 17/6290, S. 7; der hier verwendete Begriff, ,ausgleichen“ deutet eigentlich auf die Saldierung von positiven und negativen Reststimmen hin. 
allein eine Chance haben, Direktmandate zu gewinnen, Zweitstimmenergebnisse von deutlich über 40 Prozent erzielten, kam es folglich kaum zu Überhangmandaten; während von 1949 bis 1990 in zwölf Bundestagswahlen zusammen nur 23 Überhangmandate (maximal sechs pro Wahl) anfielen, waren es in den fünf Wahlen seit 1994 zusammen 74, davon allein 24 bei der Wahl 2009. ${ }^{30}$ Neben anderen Gesichtspunkten (wie etwa zunehmendes koalitionstaktisches Wahlverhalten) ist dafür in erster Linie die nachlassende Bindungskraft der (vormals großen) Volksparteien bei gleichzeitiger Vergrößerung des Parteienspektrums ursächlich; so hat keine Partei mehr in den letzten drei Bundestagswahlen 40 Prozent und in den letzten sieben Bundestagswahlen 45 Prozent der Zweitstimmen errungen. ${ }^{31}$ Dementsprechend war das öffentliche und juristische Interesse am Phänomen der Überhangmandate in den ersten Jahrzehnten der Bundesrepublik vergleichsweise gering. Dies änderte sich erst mit der Bundestagswahl 1994, bei der die Überhangmandate mit 16 erstmalig auf eine zweistellige Größe anwuchsen, die zugleich politisch bedeutsam war: Ohne diese zusätzlichen Sitze im Bundestag hätte die Regierungskoalition nämlich nur eine äußerst knappe Mehrheit (exakt die so genannte Kanzlermehrheit) erreicht, die sie erst durch den Vorsprung an acht Überhangmandaten (zwölf gegenüber vier der Oppositionsparteien) konsolidieren konnte. ${ }^{32}$ Infolge dieser Entwicklung entstand eine höchst kritische Diskussion über die Existenz der Überhangmandate, was sich auch in einem exorbitanten Hochschnellen der Einsprüche gegen das Wahlergebnis äußerte (1.453 Einsprüche, nachdem es bei den zwölf Wahlen zuvor nie mehr als 83 pro Wahl gegeben hatte). ${ }^{33}$

\section{(b) Behandlung der Überhangmandate in früheren Entscheidungen des BVerfG}

Das BVerfG hat sich vor der aktuellen Entscheidung insgesamt viermal zu Überhangmandaten geäußert; in den ersten drei Entscheidungen von 1957, 1963 und 1988 hat es das Anfallen von Überhangmandaten zwar als Erfolgswert-,,Differenzierung“ der (Zweit-)Stimmen angesehen, aber als Ausdruck des personalisierten Elements des Wahlrechts (mit seiner gewollten engen Verbundenheit zwischen Wahlkreis und direkt gewähltem Abgeordneten) „in engen Grenzen“, die gewahrt seien, akzeptiert. ${ }^{34}$ Während das Gericht bei diesen drei Entscheidungen stets nur ein bis zwei Absätze auf die Begründung verwendete (weil die Überhangmandate nicht im Zentrum standen), drehte sich im vierten Fall die ganze Entscheidung um die Frage der Zulässigkeit von Überhangmandaten. Hintergrund dafür war die erwähnte öffentliche Diskussion im Nachgang zur Bundestagswahl 1994, in deren Verlauf die niedersächsische Landesregierung unter Führung von Ministerpräsident Gerhard Schröder ein abstraktes Normenkontrollverfahren gegen die Regelung zu den Überhangman-

30 Vgl. http://www.wahlrecht.de/ueberhang/ueberhist.html (Abruf am 5. August 2012).

31 Vgl. http://www.bundestag.de/bundestag/wahlen/ergebnisse_seit1949.html (Abruf am 5. August 2012). Zu den Gründen für Überhangmandate vgl. auch BVerfG, a.a.O. (Fn. 17), Rn. 149 m.w.N.

32 Union und FDP erzielten 341 Mandate, die Oppositionsparteien 331. Zieht man die zwölf beziehungsweise vier Überhangmandate ab, verteilen sich die (damals noch 656) Ausgangsmandate zu 329 auf Union und FDP und 327 auf SPD, Bündnis 90/Die Grünen und PDS, vgl. http:// www.wahlrecht.de/ergebnisse/bundestag.htm (Abruf am 5. August 2012).

33 Vgl. Peter Schindler, Datenhandbuch zur Geschichte des Deutschen Bundestages 1949 bis 1999 , Band I, Berlin 1999, S. 298 f.

34 BVerfGE, Bd. 7, S. 63, S. 74 f., Rn. 31; BVerfGE, Bd. 16, S. 130, S. 139 f., Rn. 24 f.; BVerfGE, Bd. 79, S. 169, S. 171 f., Rn. 9. 
daten anstrengte. Weil sich der Senat damals in dieser Frage spaltete, kam es zu einer 4:4-Entscheidung, bei der gemäß $\$ 15$ Abs. 4 Satz 3 BVerfGG kein Verfassungsverstoß festgestellt werden kann, weshalb der Normenkontrollantrag abgelehnt und die Überhangmandate gebilligt wurden. Die vier diese Entscheidung tragenden Richter stellen in ihren Gründen auf die mit der Verhältniswahl verbundenen beziehungsweise vorgeschalteten „Elemente der Mehrheitswahl“ in den Wahlkreisen ab, so dass „der verhältniswahlrechtlichen Erfolgswertgleichheit aller Stimmen nur eine von vornherein begrenzte Tragweite $\mathrm{zu}$ (kommt)“ und „die Abrechnung der unmittelbar errungenen Mandate nicht stets einen vollen Ausgleich der Sitzverteilung im Sinne des Proporzes bewirken kann und soll“ ${ }^{35}$. Zwar dürfe die Zahl der Überhangmandate nicht so hoch sein, dass „sich das Wahlverfahren von den Grundentscheidungen des Gesetzes“ entferne; doch sei die quantitative Obergrenze für die Zulässigkeit von Überhangmandaten auch bei 16 (von damals 656 Ausgangsmandaten) noch nicht überschritten; als „Orientierungspunkt“ für diese Obergrenze wird die (für die Gleichheit des Erfolgswert ebenfalls kritische) Fünfprozenthürde ins Spiel gebracht: „Das Fünfprozentquorum, das dort den rechtlichen Rahmen für einen schonenden Ausgleich zwischen parteibezogener Wahlgleichheit und Funktionsfähigkeit des Parlaments bietet, mag auch hier - bezogen auf die reguläre Gesamtzahl der Parlamentssitze - als Anhalt dienen, nach dem der Abweichung von den Prinzipien der hälftigen Zusammensetzung des Bundestages nach Wahlkreis- und nach Listenmandaten und der proportionalen Verteilung der Sitze nach dem Ergebnis der für die Parteien abgegebenen (Zweit)Stimmen eine Grenze gesetzt ist. "36 Bei 598 Ausgangsmandaten wäre diese Grenze bei 30 Überhangmandaten erreicht, was angesichts der 24 bei der Wahl 2009 angefallenen Überhangmandate und der zugleich steigenden Tendenz nach dieser Entscheidung des BVerfG - erst - mittelfristig eine Reaktion des Gesetzgebers erforderlich gemacht hätte. Die vier unterlegenen Richter hingegen ziehen diese Obergrenze sehr viel enger; Überhangmandate sollen danach unzulässig sein, soweit die dadurch verbundene „Verschiebung des Gewichts der Wählerstimmen ... in ihrem Ausmaß über Unschärfen hinausgeht, welche mit jeder Sitzzuteilung im Proportionalverfahren unausweichlich verbunden sind"37.

\section{(c) Argumentation des BVerfG in seiner aktuellen Entscheidung}

In seiner aktuellen Entscheidung legt das BVerfG nun - einstimmig! - fest, dass das Wahlrecht ausgleichslose Überhangmandate nur bis zu maximal 2,5 Prozent der Ausgangsmandate (also 15 von 598) zulassen darf.

(1) Um zu diesem Ergebnis zu kommen, muss der Senat zunächst eine Zulässigkeitshürde nehmen, weil sich die verschiedenen Verfahren gegen das 19. Änderungsgesetz zum BWahlG richten, das die für die Überhangmandate verantwortliche Regelung des $\$ 6$ Abs. 5 BWahlG weder geschaffen noch inhaltlich verändert hat. Im Wege „sachgerechter Auslegung“ der Anträge erstreckt das BVerfG aber die Prüfung auf „sämtliche Regelungen des $\$ 6$ BWahlG mit Ausnahme des in Abs. 2 Satz 2 bis 7 geregelten Divisorverfahrens"; auch die bei den Verfassungsbeschwerden zu beachtende Einjahresfrist hält das BVerfG für die

35 BVerfGE, Bd. 95, S. 335, S. 356, S. 358, Rn. 89, Rn. 93.

36 Ebenda, S. 365 f., Rn. 114 f.

37 Ebenda, S. 367 f., Rn. 118. 
schon ältere Norm des $₫ 6$ Abs. 5 BWahlG gewahrt, weil die Änderungen in der BWahlGNovelle „die Bedeutung der Überhangmandate beeinflussen“38.

(2) Die inhaltliche Argumentation für die Problematisierung der Überhangmandate setzt zunächst an Aussagen der bisherigen Rechtsprechung an. So entscheide die Erststimme „grundsätzlich nur darüber ..., welche Personen als Wahlkreisabgeordnete in den Bundestag einziehen", und nicht über die (parteipolitische) Zusammensetzung des Parlaments. Weil das Bundestagswahlrecht - was verfassungsrechtlich keineswegs zwingend sei ${ }^{39}$ — „unbeschadet der Direktwahl der Wahlkreiskandidaten ... den Grundcharakter einer Verhältniswahl“ trage, müsse es „am Erfordernis der Erfolgswertgleichheit der Wählerstimmen gemessen “ 40 werden. Problematisch in diesem Zusammenhang sei die „Differenzierung des Stimmgewichts zwischen Wählern ..., deren Parteien keine Überhangmandate erzielt haben, und Wählern solcher Parteien, denen das gelungen ist“. Denn während die erstgenannten Parteien mehr (Zweit-)Stimmen benötigten, um einen Sitz im Deutschen Bundestag zu erringen, genügten den zweitgenannten weniger (Zweit-)Stimmen. Dies sei nur in dem Umfang hinzunehmen und gerechtfertigt, wie es nötig sei, „um das Anliegen der personalisierten Verhältniswahl zu verwirklichen“, also die enge Beziehung von 50 Prozent der Abgeordneten zu ihrem Wahlkreis. ${ }^{41}$

(3) Ausgehend von diesen Aussagen der bisherigen Rechtsprechung, an der ausdrücklich festgehalten wird, begründet der Senat sein Votum für maximal 15 ausgleichslose Überhangmandate. Das „hinreichend gewichtige“ Anliegen einer mit der Personenwahl verbundenen Verhältniswahl könne nur ,in begrenztem Umfang“ als Rechtfertigung für die Erfolgswertdifferenzierung gelten. ${ }^{42}$ Kernpunkt der Argumentation ist dabei die vom Gesetzgeber selbst getroffene Grundsatzentscheidung für ein Verhältniswahlrecht, weil die Gesamtzahl der 598 Sitze (gegebenenfalls nach Abzug von Grundmandaten) nach den Zweitstimmen verteilt wird ( $\$ 6$ Abs. 2 Satz 6 BWahlG), wovon dann in einem zweiten Schritt die direkt errungenen Mandate abgezogen werden ( $\$ 6$ Abs. 4 Satz 1 BWahlG). Diese eigene Grundsatzentscheidung des Gesetzgebers begrenze dann seinen Spielraum in der weiteren Ausgestaltung, weil er sich etwa bei der Erfolgswertdifferenzierung durch ausgleichslose Überhangmandate noch „innerhalb des gesetzgeberischen Konzepts halten “ ${ }^{43}$ müsse. Um für die Zukunft Rechtssicherheit zu schaffen (insbesondere für Wahlprüfungsverfahren), „hält der Senat es für geboten, die vorliegenden gesetzlichen Wertungen in einem handhabbaren Maßstab zusammenzuführen, an den der Gesetzgeber anknüpfen kann“; dieser Maßstab liege bei „etwa einer halben Fraktionsstärke“ ${ }^{44}$. In ungewohnter Offenheit räumt das BVerfG ein,

38 BVerfG, a.a.O. (Fn. 17), Rn. 45, Rn. 49.

39 Das BVerfG (ebenda) bekennt sich ausdrücklich erneut zum Gestaltungsspielraum des Gesetzgebers, der sich auch für ein Mehrheitswahlrecht, Grabenwahlrecht oder ähnliches entscheiden könne (Rn. 56). In der Literatur gibt es aber auch Stimmen, die nur das Verhältniswahlrecht als „verfassungsadäquat" ansehen, vgl. Hans-Heinrich Trute, in: Ingo von Münch / Philip Kunig (Hrsg.), Grundgesetz-Kommentar, Band 2, München 2001, Art. 38 Rn. 15. Dem widerspricht Hans H. Klein, a.a.O. (Fn. 1), Art. 38 Rn. 122, wonach „ein verfassungsrechtlicher Mehrwert ... der Verhältniswahl nicht zu(komme)".

40 BVerfG, a.a.O. (Fn. 17), Rn. 112, Rn. 116.

41 Ebenda, Rn. 117, Rn. 125.

42 Ebenda, Rn. 132.

43 Ebenda, Rn. 127, Rn. 137.

44 Ebenda, Rn. 140. 
„dass die Zahl von 15 Überhangmandaten als Akt richterlicher Normkonkretisierung nicht vollständig begründet“ werden kann; auch sei die Festlegung der Grenze „,in erster Linie der politischen Willensbildung im Gesetzgebungsverfahren“ vorbehalten, doch sei es ,im speziellen Zusammenhang Aufgabe des Bundesverfassungsgerichts, gleichheitsrechtliche Anforderungen ... so zu konkretisieren, dass der Gesetzgeber das Wahlrecht auf verlässlicher verfassungsrechtlicher Grundlage gestalten kann" und die Gefahr einer Bundestagsauflösung wegen eines verfassungswidrigen Wahlrechts „minimiert“ werde. ${ }^{45}$

(4) Ausdrücklich wendet sich der Senat gegen die Äußerung der vier die Entscheidung von 1997 tragenden Richter, wonach die Fünfprozenthürde hier einen Anhaltspunkt bieten könne; er betont, dass für diese Zugangsschranke Rechtfertigungsgründe maßgeblich seien (Funktionsfähigkeit des Parlaments), auf die es bei der Frage nach Überhangmandaten gar nicht ankomme. Zudem, so das Gericht, sei in der Entscheidung kein Zahlenwert festgelegt, sondern nur ein möglicher Orientierungswert genannt worden. ${ }^{46}$ Schließlich sei die jetzt festgelegte Größenordnung auch noch mit der Entscheidung von 1997 kompatibel, bei der 16 Überhangmandate von 656 Ausgangsmandaten gebilligt worden seien.

(5) Von politischem Interesse - nicht zuletzt für die jetzt anstehenden Neuverhandlungen der Fraktionen über das 20. Änderungsgesetz zum BWahlG - sind auch noch die kritischen Anmerkungen des Gerichts zu der im Vorfeld diskutierten Überlegung, die Überhangmandate durch Ausgleichsmandate für die anderen Parteien politisch zu kompensieren. Aus Karlsruher Sicht würde eine solche Lösung wegen der damit verbundenen „nicht vorhersehbaren Erhöhung der Sitzzahl des Bundestages“ zu „Praktikabilitätsproblemen“ sowie „Beeinträchtigungen des föderalen Proporzes“ führen und das gesetzgeberische Ziel einer hälftigen personenbezogenen Legitimation der Abgeordneten gefährden. ${ }^{47}$

\section{(d) Bewertung}

Die Passagen zu den Überhangmandaten sind fraglos der „politischste " und problematischste Teil der Entscheidung, denn das BVerfG macht hier zwei Dinge, die es sonst peinlich zu vermeiden bemüht ist: Zum einen bricht es offen mit der bisherigen Rechtsprechungslinie in einer politisch bedeutsamen und verfassungsrechtlich sensiblen Frage und zum anderen reduziert es dabei den gesetzgeberischen Ermessensspielraum auf Null. Zur Rechtfertigung bedient sich der Senat einer wesentlich mehr politisch als juristisch anmutenden Begründung.

(1) Es ist bemerkenswert, wie sich der Senat über die Entscheidung von 1997 hinweggesetzt hat. Das wird auch nicht substanziell dadurch geschmälert, dass die seinerzeitige Entscheidung lediglich mit 4:4 Stimmen erging, denn letztlich kann nur maßgeblich sein, was Aussage einer getroffenen Entscheidung - mit welcher Mehrheit auch immer - war. Ebenfalls schwach ist der Einwand, es läge gar kein Bruch mit der Entscheidung von 1997 vor, weil sich die damals gebilligten 16 Überhangmandate innerhalb der jetzt im Tenor genannten Quote bewegen: Gerade die Entscheidungen des BVerfG haben meistens eine weit über ihren Entscheidungstenor hinausreichende Bedeutung, weil in den Gründen oft grundsätzliche verfassungsrechtliche Fragen geklärt werden (teilweise über den Entscheidungsgegen- 
stand hinaus, was schon zu Kritik geführt hat). Deshalb war die Aussage der Entscheidung von 1997 nicht in erster Linie die, dass 16 Überhangmandate akzeptabel sind, sondern dass bei einer Annäherung an die Fünfprozentgrenze die Handlungspflicht des Gesetzgebers zur Begrenzung der Überhangmandate ausgelöst wird. Daran ändert auch die terminologische Einordnung als „Orientierungswert“ nichts. Auch wenn dem Gericht darin zuzustimmen ist, dass die Überhangmandate de lege lata tendenziell noch weiter zunehmen würden und mit 24 Mandaten bei der letzten Wahl die Grenze nicht mehr weit entfernt ist, konnte man auf der Basis der bisherigen Rechtsprechung lediglich einen mittelfristigen Änderungsbedarf konstatieren. Eine Nichtigkeitserklärung der bisherigen Regelung war dagegen zum gegenwärtigen Zeitpunkt in keiner Weise zu erwarten, nicht zuletzt vor dem Hintergrund des quasi ausdrücklichen Schweigens des Gerichts in der Wahlrechtsentscheidung von 2008. Insofern bricht das BVerfG unbeschadet der „klassischen“ argumentativen Einbettung der Problematik in diesem konkreten Punkt in einer überraschenden Weise mit der bisherigen Rechtsprechungslinie, was im Hinblick auf das hohe Gut der Verlässlichkeit und Berechenbarkeit höchstrichterlicher Rechtsprechung äußerst dysfunktional ist. Das mag in der Sache diejenigen (bedingt) freuen, die mit den Überhangmandaten schon immer auf Kriegsfuß standen. Diejenigen aber, die auf den Fortbestand der Rechtsprechungslinie vertraut haben, dürfen zu Recht enttäuscht sein. Zu letzteren gehört offensichtlich auch der (mehrheitliche) Wahlrechtsgesetzgeber.

(2) Nicht minder ungewöhnlich ist der vom Gericht selbst so bezeichnete „Akt richterlicher Normkonkretisierung", und zwar in zweierlei Hinsicht:

Erstens wird man dem BVerfG eine ganz erhebliche Konkretisierungsleistung attestieren dürfen: Um den Grundsatz der gleichen Wahl (Art. 38 Abs. 1 GG) in Verbindung mit der Grundsatzentscheidung des Gesetzgebers für ein schwerpunktmäßiges Verhältniswahlrecht auf die Zahl von gerade maximal 15 zulässigen Überhangmandaten herunter zu brechen, bedarf es schon einer besonderen Norminterpretationskunst. Im Sinne einer politischen Entscheidungslogik wäre die Zahl einfach erklärbar: Wenn (wie 1997) die eine Hälfte für ein Festhalten an den 30 Überhangmandaten und die andere Hälfte für eine völlige Abschaffung der (ausgleichslosen) Überhangmandate gewesen wäre, würde die Zahl 15 den klassischen Kompromiss darstellen. Damit wäre die 4:4-Spaltung zugunsten einer überzeugenden Einstimmigkeit vermieden, zumal die Quote von 2,5 Prozent gerade der zuletzt (1997) gebilligten Mandatszahl (16 von 656) entspricht. Da aber die Karlsruher Richter regelmäßig betonen, dass sie sich als Gericht - und nicht als politisches Organ - verstehen ${ }^{48}$, erstaunt umso mehr die Offenheit, mit der die Unmöglichkeit einer „vollständigen Begründung“ eingeräumt wird.

Dies führt zum zweiten Punkt: Was juristisch nicht begründet werden kann, ist letztlich politisch motiviert. Und wie das Gericht selbst so richtig feststellt, obliegen politische Entscheidungen dem Gesetzgeber. Trifft es zu, wie oft betont wird, dass der Gesetzgeber einen weiten Gestaltungsspielraum hat, dürfte dieser nicht ohne bedenkliche Verschiebungen im Gewaltenteilungssystem gerichtlich „enggeführt“ oder - wie in diesem Fall - sogar auf Null

48 Vgl. die Aussagen des Präsidenten des Bundesverfassungsgerichts Andreas Voßkuhle beim Wissenschaftsforum des Deutschen Bundestages am 17. November 2011, abgedruckt in: Unjuristische und unpolitische Argumente: Ein Disput zweier Präsidenten von Verfassungsorganen, in: FAZ vom 22. Dezember 2011, S. 30; Günter Bannas, Gesetzgebende und rechtsprechende Gesetzgeber, in: FAZ vom 18. November 2011, S. 7. 
reduziert werden. Leider erliegt das BVerfG hier (nicht zum ersten $\mathrm{Mal}^{49}$ ) der Versuchung, sich als Gesetzgeber an die Stelle des Parlaments zu setzen - gemäß der Devise: „Wenn Not am Mann ist, dann machen wir es auch selbst. " ${ }^{50}$ Die fürsorgliche Argumentation, damit Rechtssicherheit für künftige Bundestagswahlen zu schaffen, ist relativ schwach: Hätte das BVerfG ohne Festlegung einer konkreten Zahl deutlich gemacht, dass es an der Fünfprozentquote von 1997 nicht mehr festhält und eine geringere Zahl für geboten hält, hätte der Bundestag noch einen gewissen Spielraum gehabt, den dann das BVerfG wegen des hohen Bestandsschutzes einer gewählten Volksvertretung auch im Wahlprüfungsverfahren hätte respektieren können (wie es auch 2008 aus guten Gründen nicht nur auf eine Aufhebung der Wahl von 2005 verzichtete, sondern sogar noch eine weitere Wahl nach verfassungswidrigem Wahlrecht in Kauf nahm). Konsequent zu Ende gedacht würde die Fürsorge-Argumentation bedeuten, dass letztlich alle wichtigen Gesetzesentscheidungen zur Vermeidung des Verdikts der Verfassungswidrigkeit gleich vom BVerfG konkret vorgezeichnet werden müssten. Auch der Umstand, dass das Wahlrecht beim Parlament (neben Diäten und Parteienfinanzierung) zur „Gesetzgebung in eigener Sache“ zählt, rechtfertigt keine richterliche Rechtsetzung, sondern nur eine strenge Missbrauchskontrolle. ${ }^{51}$ Diese ist jedoch bei den Überhangmandaten nicht besonders relevant: Ein Blick auf ihre Geschichte zeigt, dass sie nie mehrheitsbegründend, sondern in der Regel mehrheitsverstärkend gewirkt haben ${ }^{52}$; dabei handelt es sich um keinen Zufall, denn die relativ stärkste politische Kraft gewinnt auch regelmäßig den größeren Teil der Direktmandate. Da die Mehrheiten bei den verschiedenen Bundestagswahlen unterschiedlich ausgefallen sind, haben davon die beiden großen Volksparteien proportional anteilig zu ihren Wahlsiegen profitiert. So haben seit 1949 in 17 Bundestagswahlen die Unionsparteien 62 und die SPD 34 Überhangmandate errungen, was ziemlich genau der jeweiligen Quote an Kanzlerjahren entspricht (von 1949 bis 2012 haben die Unionsparteien 43 und die SPD 20 Jahre die Regierungsspitze besetzt). Auch gegenüber

49 Das Thema ist keineswegs neu, vgl. zum Beispiel Philipp Austermann, Die rechtlichen Grenzen des Bundesverfassungsgerichts im Verhältnis zum Gesetzgeber, in: DÖV 2011, 267; Andreas Götz I Lina Schneider, Das Bundesverfassungsgericht als Ersatzgesetzgeber - Methodische Bemerkungen zu dem Urteil des Bundesverfassungsgerichts vom 7. September 2011 in Sachen Finanzhilfen für Griechenland und Euro-Rettungsschirm, in: DVBl. 2012, S. 145; eine angemessene Zurückhaltung bescheinigt dem BVerfG Christopher Klotz, Die Machtbalance zwischen Politik und verfassungsgerichtlicher Rechtsprechung, in: ZRP 2012, S. 5.

50 Äußerung von Andreas Voßkuhle angesichts der Probleme des Gesetzgebers, fristgerecht das neue Wahlrecht zu verabschieden, siehe http://www.zeit.de/politik/deutschland/2011-09/wahlrechtverfassungsgericht (Abruf am 7. August 2012).

51 Vgl. die Problemanalyse von Hans Herbert von Arnim, Wahlgesetze: Entscheidungen des Parlaments in eigener Sache, in: JZ 2009, S. 813.

52 Allein 1983 hat nur die Opposition (zwei) Überhangmandate errungen, vgl. http://www.wahlrecht.de/ueberhang/ueberhist.html (Abruf am 5. August 2012). Siehe auch Joachim Behnke / Florian Grotz, Das Wahlsystem zwischen normativer Begründung, empirischer Evidenz und politischen Interessen. Ein Kommentar zu Gerd Strohmaier sowie Franz Urban Pappi und Michael Herrmann, in: ZParl, 42. Jg. (2011), H. 2, S. 419 - 425, S. 422. Im griechischen Wahlrecht ist diese mehrheitsverstärkende Wirkung sogar - bei einem Verhältniswahlrecht! - mit einem Bonus von 50 Sitzen für die relativ stärkste Partei ausdrücklich vorgesehen, was bei den Wahlen 2012 angesichts der Schwäche der dortigen Parteien beinahe eine Verdoppelung der Mandate für die betroffene Partei bedeutet hat, vgl. http://www.wirtschaftsblatt.at/home/international/wirtschaftspolitik/griechisches-wahlrecht-50-bonus-mandate-fuer-den-sieger-517168/index.do (Abruf am 7. August 2012). 
den „kleineren“ Parteien stellt das Instrument der Überhangmandate keinen Missbrauch dar, weil es im Vergleich zu dem laut Karlsruhe ebenfalls möglichen Mehrheits- oder Grabenwahlrecht das wesentlich mildere Mittel darstellt.

(3) Aber noch weitergehender lässt sich fragen, ob der Gesetzgeber wirklich - wie das BVerfG in ständiger Rechtsprechung betont - dazu verpflichtet ist, auf eine Begrenzung der Überhangmandate zu achten. Basis der auf diese Verpflichtung zielenden Argumentation ist das vom BVerfG faktisch aufgestellte „Gebot zur Typenklarheit“ im Wahlrecht. So unterstreicht das Gericht mehrfach, dass der Gesetzgeber, entscheidet er sich für das Verhältniswahlrecht, dies auch konsequent durchhalten müsse. Doch ist das wirklich richtig? Nicht zum ersten Mal betonen die Karlsruher Richter in dieser Entscheidung, dass sich der Gesetzgeber auch für ein Mehrheits- oder Grabenwahlrecht entscheiden könnte. Bei Letzterem handelt es sich um ein paritätisches Mischmodell von Mehrheits- und Verhältniswahl, bei dem jeweils 299 Sitze nach relativer Wahlkreis-Mehrheit (Erststimme) und nach Verhältniswahl zwischen den Parteilisten (Zweitstimme) vergeben würden. Nun hat der Bundestag noch einen Schritt weiter in Richtung Verhältniswahlrecht getan, indem er in einem ersten Schritt alle 598 Sitze nach dem Zweitstimmenproporz vergeben will; gleichzeitig aber hat er mit der eigenständigen Erststimme ein klares Element des Mehrheitswahlrechts vorgesehen, das sich im Fall der Überhangmandate auch gewollt und außerhalb des (Zweitstimmen-) Proporzes auf die Sitzverteilung auswirkt. Insofern haben wir - was auch Karlsruhe anerkennt - kein reines, aber eben auch kein „grundsätzliches“ Verhältniswahlrecht, sondern ein Mischmodell mit (überwiegenden) Verhältnis- und (geringeren) Mehrheitswahlrechtselementen. ${ }^{53}$ Dann muss aber auch dieses Mischmodell als gesetzgeberisches Grundkonzept akzeptiert werden mit der Folge, dass die Frage der Erfolgswertgleichheit nur insoweit nach Proporzgesichtspunkten beurteilt werden darf, wie das Mehrheitswahlelement nicht zum Tragen kommt (was bei den Überhangmandaten eben der Fall ist). Insofern ist es in allererster Linie keine verfassungsrechtliche, sondern eine politische Entscheidung des Gesetzgebers, ob und in welchem Umfang Überhangmandate vorgesehen sein sollen und ob es dafür eine Begrenzung oder Ausgleichsmandate geben soll.

\section{Fazit: Eingriff in die Gestaltungsfreiheit des Gesetzgebers}

Die aktuelle Wahlrechtsentscheidung des BVerfG lässt die - auch in der mündlichen Urteilsverkündung angeklungene - ${ }^{54}$ Verärgerung des Gerichts darüber spüren, dass der Wahlrechtsgesetzgeber trotz einer großzügig bemessenen Umsetzungsfrist keinen durchgreifenden Ansatz einer Neuregelung gefunden hat, sondern sich auf einen „minimalinvasiven Eingriff“ beschränkt hat. Eventuell hat auch die - in Wahlrechtsfragen ungewöhnliche - parteipolitische Dichotomie zwischen Regierungs- und Oppositionslager dazu beigetragen. Es drängt

53 Wie hier: Franz Urban Pappi / Michael Herrmann, a.a.O. (Fn. 1), S. 271; gegen ein Verständnis als „Mischwahlsystem“ Gerd Strohmeier, Die Geister, die Karlsruhe rief - eine Replik auf die Beiträge zur Wahlsystemreform in Heft 2/2010 der ZParl, in: ZParl, 42. Jg. (2011), H. 1, S. 186 193; Joachim Behnke / Florian Grotz, a.a.O. (Fn. 52), S. 420 f.

54 Trotz einer „großzügig bemessenen, dreijährigen Frist für den Wahlgesetzgeber, eine verfassungsgemäße Neuregelung zu treffen, ist das Ergebnis - das ist übereinstimmende Auffassung im Senat - ernüchternd“", so Präsident Andreas Voßkuhle bei der mündlichen Urteilsbegründung, vgl. http:// www.tagesschau.de/inland/wahlrechtsreform114.html (Abruf am 7. August 2012). 
sich jedenfalls der Eindruck auf, dass es bei der Aufgabenteilung zwischen (Verfassungs-) Rechtsprechung und Gesetzgebung bezüglich der Entscheidungskompetenz über die Reichweite und den Inhalt eines Regelungsansatzes im Wahlrecht zu einer Verschiebung gekommen ist.

Karlsruhe greift mit dieser Entscheidung jedenfalls weitreichend in die regelmäßig postulierte Gestaltungsfreiheit des Gesetzgebers ein. Soweit dies den Effekt des negativen Stimmgewichts betrifft, überzeugt die gerichtliche Behauptung einer nicht reduzierten Wahrscheinlichkeit für den Eintritt dieses Effekts nicht; vielmehr hat das Parlament diese Wahrscheinlichkeit - orientiert an der politischen Lebenswirklichkeit - erheblich reduziert, so dass das Verdikt der zur Nichtigkeit führenden Verfassungswidrigkeit nicht zwingend gewesen wäre. Anders verhält es sich mit der Berechnung der neu geschaffenen Zusatzmandate, die ausschließlich auf Rundungsverlusten (also positiven Reststimmen) beruht. Dadurch wird eine Partei, die mehr positive Reststimmen aufbringt, gegenüber einer anderen mit weniger positiven Reststimmen jedenfalls dann ohne Sachgrund bevorzugt, wenn beide Parteien eine ähnliche Gesamtbilanz bei Verrechnung der positiven und negativen Reststimmen haben. Allerdings betrifft dieser Verfassungsverstoß für sich genommen nur einen abgrenzbaren Teil des Wahlrechts, nämlich die Berechnung und Zuteilung von Zusatzmandaten gemäß $₫ 6$ Abs. 2a BWahlG. Die gerichtliche Festlegung einer konkreten Höchstzahl an zulässigen ausgleichslosen Überhangmandaten stellt einen fürsorglichen, aber unter dem Gesichtspunkt der Gewaltenteilung äußerst fragwürdigen Übergriff des BVerfG in die Domäne des Gesetzgebers dar; nach der hier vertretenen Auffassung wird die gesamte Behandlung der Überhangmandate dem gesetzgeberischen Konzept des Wahlrechts nicht gerecht, weil dieses als grundsätzliches Verhältniswahlrecht gesehen wird, obwohl es ein Mischsystem aus überwiegenden Verhältniswahl- und (in geringerem Umfang) Mehrheitswahlelementen darstellt. Würde man das Wahlrecht so akzeptieren, wie es konzipiert ist, wäre die Erfolgswertgleichheit auf die Zweitstimmen beschränkt, soweit nicht das Mehrheitswahlelement etwa in Gestalt der Überhangmandate zum Tragen käme. Dann aber wären die Überhangmandate keine Verletzung der Erfolgswertgleichheit, sondern Ausdruck eines eigenständigen Teilelements des Wahlrechts.

Insofern wäre es verfassungsrechtlich gut vertretbar gewesen, dem Parlament seine politische Verantwortung zu belassen und unter Beherzigung des leider immer mehr in Vergessenheit geratenen Grundsatzes des ,judicial self restraint“ die Nichtigkeitserklärung auf die Regelung über die Zusatzmandate zu beschränken. So aber hat das BVerfG den Weg in die weitere Politisierung des Gerichts und Verrechtlichung der Politik gewählt, weshalb „am Ende ... Abgeordnete wie Verfassungsrechtler (argumentieren) und Verfassungsrichter ...

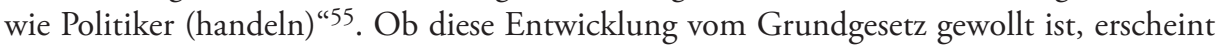
doch sehr fraglich.

55 Christine Landfried, Jedem das Seine, in: FAZ vom 26. Juli 2012, S. 8. Zu optimistisch daher Bernd Rüthers, Klartext zu den Grenzen des Richterrechts, in: NJW 2011, S. 1856. Siehe auch Joachim Jan, Gerichte sollen den Gesetzgeber nicht länger entmündigen, in: FAZ vom 9. März 2011, S. 21 (ausgelöst allerdings vom EuGH). 